\title{
Aldosteronism and Resistant Hypertension
}

\author{
Maria Czarina Acelajado' ${ }^{1}$ and David A. Calhoun ${ }^{1,2}$ \\ ${ }^{1}$ Vascular Biology and Hypertension Program, University of Alabama at Birmingham, CH19, Room 115, \\ 1530 3rd Avenue South, Birmingham, AL 35294-2041, USA \\ ${ }^{2}$ Division of Cardiovascular Disease, Department of Medicine, University of Alabama at Birmingham, AL 35294-2041, USA
}

Correspondence should be addressed to Maria Czarina Acelajado, czarina.md@gmail.com

Received 3 October 2010; Revised 3 December 2010; Accepted 10 December 2010

Academic Editor: Konstantinos Tsioufis

Copyright ( $) 2011$ M. C. Acelajado and D. A. Calhoun. This is an open access article distributed under the Creative Commons Attribution License, which permits unrestricted use, distribution, and reproduction in any medium, provided the original work is properly cited.

\begin{abstract}
Resistant hypertension (RHTN) is defined as blood pressure (BP) that remains uncontrolled in spite of intake of $\geq 3$ antihypertensive medications, ideally prescribed at optimal doses and one of which is a diuretic. The incidence of primary aldosteronism (PA) in patients with RHTN is estimated in prospective studies to be 14 to $23 \%$, which is higher than in the general hypertensive population. Patients with PA are at an increased cardiovascular risk, as shown by higher rates of stroke, myocardial infarction, and arrhythmias compared to hypertensive individuals without PA. Likewise, RHTN is associated with adverse cardiovascular outcomes, and the contribution of PA to this increased risk is undetermined. Similar to PA, obstructive sleep apnea (OSA) is closely associated with RHTN, and a causal link between PA, OSA, and RHTN remains to be elucidated. The addition of MR antagonists to the antihypertensive regimen in patients with RHTN produces a profound BP-lowering effect, and this effect is seen in patients with or without biochemical evidence of PA, highlighting the role of relative aldosterone excess in driving treatment resistance in this group of patients.
\end{abstract}

\section{Introduction}

Primary aldosteronism (PA) is characterized by the overproduction of the mineralocorticoid hormone aldosterone by the adrenal gland, a condition that is relatively autonomous of the renin-angiotensin system (RAS), and nonsuppressible by sodium loading [1]. The elevated circulating aldosterone levels lead to potassium loss, hypernatremia, metabolic alkalosis, and hypertension. The syndrome can be the result of bilateral hyperplasia of the adrenal glands, unilateral adrenal hyperplasia, aldosterone producing adrenal adenoma, or in rare cases, by glucocorticoid-remediable aldosteronism (GRA).

Early epidemiologic studies have determined the prevalence of PA to be $<1 \%$ of hypertensive patients, particularly if hypokalemia was used as a sine qua non for its diagnosis [2-4]. Recent studies have challenged this assumption, which have reported the prevalence of PA to be about 5-10\% in the general hypertensive population [5-8]. In a study done in Chile, using the aldosterone to renin ratio (ARR) to screen for PA and confirming its presence using fludrocortisone testing, the prevalence of PA was $6.1 \%$ in a population of unselected essential hypertensive patients [8]. This prevalence was noted to rise with increasing severity of hypertension, such that in patients with Stage III hypertension (classified according to Joint National Commission (JNC) 6 criteria, i.e., $\mathrm{BP}>180 / 100 \mathrm{mmHg}$ ), the prevalence of PA was $13.2 \%$, as opposed to $1.99 \%$ in patients with Stage I hypertension (systolic BP [SBP] 140-159 mmHg, diastolic BP [DBP] 90$99 \mathrm{mmHg})$. Further, patients with PA had a higher BP $(164 / 102 \pm 12 / 10 \mathrm{mmHg})$ than patients without PA (156/96 \pm $16 / 9 \mathrm{mmHg}, P<.05)$ and were taking more antihypertensive medications at baseline $(1.6 \pm 0.8$ versus $1.2 \pm 0.9, P<$ $.05)$. In another study done at the Czech Republic involving over 400 patients with moderate to severe hypertension (BP > 166/101 mmHg), the prevalence of PA was 19\% [9]. In this study, screening for PA was undertaken using the ARR and confirmed by non- or mild suppression of plasma aldosterone levels after saline infusion, and the elevated BP levels in the clinic were confirmed by 24-hour ABPM.

$\mathrm{PA}$ is particularly common in patients with resistant hypertension (RHTN), defined as BP that remains above goal 
in spite of use of at least 3 antihypertensive medications, ideally prescribed at optimal doses and one of which is a diuretic [10]. In a large retrospective study involving 1,616 patients with RHTN who were referred to a specialty clinic in Greece, the prevalence of PA (screened for using the aldosterone-to-renin ratio [ARR] and confirmed by intravenous saline loading or fludrocortisone suppression and further confirmed by assessing response to spironolactone monotherapy) was determined to be $11.3 \%$ [11]. Prospective studies have found an even higher prevalence of PA in patients with RHTN. Among 88 patients with RHTN who were consecutively referred to the hypertension clinic of the University of Alabama at Birmingham (UAB), 18 patients (20\%) were confirmed to have PA, based on a high 24hour urinary aldosterone excretion $(>12 \mu \mathrm{g} / 24 \mathrm{hr})$ paired with a suppressed plasma renin activity level $(<1 \mathrm{ng} / \mathrm{ml} / \mathrm{hr})$ during a high sodium diet (urinary sodium excretion $>200 \mathrm{mEq} / 24 \mathrm{hr}$ ) [12]. This high prevalence of PA in patients with moderate to severe hypertension has been confirmed in other prospective studies done elsewhere. Investigators from Oslo, Norway found a PA prevalence of $21 \%$ of patients with RHTN [13]. In a university-based hypertension clinic in Seattle, Washington, the PA prevalence was $17 \%$ in patients with severe and/or poorly controlled hypertension (BP > 140/90 mmHg) [14]. Lastly, in a study done on 100 diabetic patients with RHTN, PA was confirmed in $14 \%$ of the study patients, using oral or intravenous saline loading [15]. Overall, these studies show that PA prevalence is higher (14-21\%) in patients with moderate to severe hypertension on multiple antihypertensive medications, compared to the general hypertensive population.

\section{Prognosis}

High aldosterone levels lead to increased sodium and water reabsorption (and consequently potassium loss) in the distal nephron, acting via mineralocorticoid receptors (MRs) that regulate gene transcription. Aldosterone also exerts rapid, nongenomic cellular effects on MRs found in nonepithelial tissue, thereby influencing cell volume, oxidation-reduction state, and vascular function [16]. These effects are linked to the development of vascular stiffness and fibrosis, particularly in large arteries, the heart, and the kidney [17]. Taken together, these effects act in concert to raise BP and contribute to target organ damage in hypertensive individuals with aldosterone excess and may partly explain treatment resistance in those with more severe hypertension.

Aldosterone excess has been linked to the development and progression of several cardiovascular diseases, notably hypertension, congestive heart failure, coronary artery disease, chronic kidney disease, and stroke. In the Framingham Offspring Study, higher baseline serum aldosterone levels were associated with an increased risk of BP elevation or development of hypertension after four years in 1688 normotensive individuals (mean age 55 years, 58\% women) [18]. Using multivariate analysis, there was a $16 \%$ increased risk of an elevation in $\mathrm{BP}(P=.002)$ and a $17 \%$ increased risk of development of hypertension per quartile increment in the serum aldosterone level, and the highest quartile of serum aldosterone, compared to the lowest quartile, was associated with a 1.61-fold risk of development of hypertension $(95 \%$ CI 1.05 to 2.46 ).

Beyond its effects on BP, the presence of PA is associated with increased carotid intima thickness, higher pulse wave velocity (a marker of increased arterial stiffness), and impaired flow-mediated brachial artery dilation (which is an indicator of endothelial dysfunction) compared to hypertensive patients without PA $[19,20]$. Left ventricular wall thickness is higher, and there is greater incidence of diastolic dysfunction as measured by tissue Doppler imaging in patients with PA compared to matched hypertensive controls [21]. In the kidney, apart from its effects on renal sodium handling, excess aldosterone induces early kidney damage. Patients with PA have a higher urinary albumin excretion rate compared to matched hypertensive controls, with a preserved glomerular filtration rate (which is an early manifestation of kidney disease) [22].

Comparing hypertensive patients with PA to those without biochemical evidence of aldosterone excess, the former had a greater incidence of stroke (both hemorrhagic and ischemic, odds ratio [OR] 4.2, 95\% confidence interval [CI] 2.0 to $8.6, P<.001$ ), myocardial infarction (OR 6.5, $95 \%$ CI 1.5 to $27.4, P<.005$ ), and atrial fibrillation (OR 12.1, 95\% CI 3.2 to $45.2, P<.0001$ ) [23]. Similarly, in another study, the presence of PA was associated with a higher incidence of myocardial infarction, stroke or transient ischemic attack, and sustained arrhythmias compared to patients with essential hypertension without PA [24]. In both studies, the differences were noted in spite of similar BP levels between the two groups, further supporting the concept that aldosterone excess produces additional adverse cardiovascular effects independent of BP. Moreover, the subtype of PA (aldosterone producing adenoma or bilateral adrenal hyperplasia) did not appear to influence the cardiovascular risk.

In the same regard, patients with RHTN have greater cardiovascular risk compared to those who do not have RHTN. Patients with RHTN have a higher incidence of concomitant diabetes, kidney disease, and obstructive sleep apnea, all of which are independent risk factors for the development of cardiovascular disease $[25,26]$. RHTN is associated with the increased incidence of left ventricular hypertrophy, retinopathy, nephropathy, and carotid intimal disease, which are considered signs of target organ damage in hypertensive disease [27, 28]. Lastly, small outcome studies have shown that patients with RHTN have increased rates of stroke, myocardial infarction, and congestive heart failure compared to patients without RHTN $[29,30]$. The extent to which PA contributes to this increased risk in patients with RHTN is unknown.

\section{Primary Aldosteronism, Obstructive Sleep Apnea, and Resistant Hypertension}

Obstructive sleep apnea (OSA) is strongly associated with the risk of having hypertension and the risk of developing 
hypertension [31]. Moreover, it appears that greater severity of hypertension is associated with increased risk of OSA, such that OSA is particularly common in patients with RHTN. In a prospective study on 41 patients with RHTN, 83\% were diagnosed with OSA (defined as apnea-hypopnea index [AHI] > 10 events/hour) after overnight polysomnography (PSG), and OSA was more prevalent (96 versus $65 \%, P=$ .014 ) and more severe (mean AHI 32 versus $14, P=0004$ ) in men than in women [25]. These results were confirmed in our study involving 71 patients with RHTN, where the prevalence of OSA was determined to be $85 \%$ and is also more common and more severe in men than in women [32].

Increasing severity of OSA also is associated with difficulty to control hypertension. In 257 patients with OSA who are adherent to a stable antihypertensive regimen for 6 months, those who were ineffectively treated (BP > $140 / 90 \mathrm{mmHg}$ ) had a higher AHI (44 events/hr) compared to those with controlled BP (33 events/hr, $P<.005)$ in spite of having similar nocturnal oxygenation and after adjusting for age, gender, and body mass index [33]. As a corollary, in an observational study on patients with RHTN and OSA, treatment of OSA with continuous positive airway pressure (CPAP) allowed deescalation of antihypertensive drug therapy (by dose reduction or discontinuation of one or more drugs) in $71 \%$ of study patients [34]. This effect was not seen in patients without RHTN. The results of a prospective study in patients with RHTN and OSA largely confirm these findings. Treatment with CPAP (mean use of 5.8 hours/night) on top of the usual antihypertensive drug regimen significantly reduced mean 24 -hour BP by $10 / 7 \mathrm{mmHg}$ at the 3rd month followup, and the BP lowering effect was greatest for those who used CPAP longest each night (i.e., better compliance) [35]. While OSA is a known risk factor for the development of hypertension and likely contributes to treatment resistance, the above results also suggest that the reverse may be true; that RHTN may predispose to or aggravate OSA. Their common link, however, remains to be elucidated.

In 325 newly diagnosed hypertensive patients who were screened for the presence of OSA and evaluated by overnight polysomnography, 53 patients were confirmed to have OSA [36]. Out of these 53 patients, 18 patients had $\mathrm{PA}$, based on results of saline infusion testing. Considering that both PA and OSA are common in patients with RHTN, it is interesting to note that in 71 patients with RHTN who underwent biochemical testing for PA and overnight PSG, the plasma aldosterone concentration was positively correlated with AHI (Pearson's $r=0.44, P=$ .0002) [32]. This association was not seen in 29 control patients without RHTN, where the median plasma aldosterone level was lower than in patients with RHTN and not correlated to the AHI. These results suggest that in patients with RHTN, PA may contribute to increased severity of OSA but does not exclude the reverse: that OSA stimulates aldosterone release in these patients. Currently, we are undertaking a prospective evaluation to test this hypothesis.

\section{Treatment}

Surgical treatment (unilateral adrenalectomy) should be offered to patients with unilateral adrenal disease who are eligible for surgery. In a number of reports, unilateral adrenalectomy has been shown to improve BP control and incidence of hypokalemia. In patients who are unable or unwilling to undergo surgery, treatment with an MR antagonist should be initiated.

Treatment of aldosterone excess with either spironolactone or unilateral adrenalectomy was found to reverse the increase in cardiovascular risk found in hypertensive patients with PA [23]. After a 12-year followup, patients with PA who were treated accordingly had similar rates of cardiovascular events as hypertensive patients without PA. Better cardiovascular outcomes were seen in younger patients and in those with a shorter duration of disease, highlighting the importance of early recognition and treatment to reverse the adverse effects of excess aldosterone.

4.1. Mineralocorticoid Receptor Antagonists. Spironolactone, a direct antagonist of the MR, is a formidable add-on agent to the antihypertensive regimen in patients with RHTN, who are taking at least 3 antihypertensive medications to control $B P$. When added to a regimen that typically already includes a diuretic, an RAS blocker and a CCB or BB, spironolactone (at a dose of 12.5 to $25 \mathrm{mg} /$ day) lowers BP by as much as $21 / 10 \pm$ $21 / 14 \mathrm{mmHg}$ at 6 weeks and 25/12 $\pm 20 / 12 \mathrm{mmHg}$ after 6 months (Figure 1) [37]. This effect was similar in patients with or without evidence of aldosterone excess, as well as in African American and Caucasian patients. In another study, incorporating the results of 24-hour ambulatory blood pressure monitoring, higher doses of spironolactone (25 to $100 \mathrm{mg} /$ day) reduced 24-hour mean BP by $16 / 9 \mathrm{mmHg}$, and control (daytime BP $<135 / 85$ ) was achieved in $48 \%$ of patients [38]. In both studies, similar degrees of BP reduction were achieved regardless of baseline plasma aldosterone or PRA values. This underscores the contributory role of relative aldosterone excess to treatment resistance in this group of patients, such that even those with presumably low levels of aldosterone benefit from MR blockade.

This importance of relative aldosterone excess in promoting treatment resistance is further emphasized in a comparison between spironolactone added to an ACE inhibitor or an ARB versus the combination of an ACE inhibitor and an ARB in treating patients with RHTN [39]. In this open-label prospective crossover study, 44 patients with RHTN received a second RAS blocker (ACE inhibitor or ARB) for 12 weeks, then subsequently given spironolactone $(25 \mathrm{mg} /$ day or higher) after a 4-week washout period. Greater BP reduction was achieved when spironolactone was added to an ACE inhibitor or an ARB (24-hour mean BP reduction of $21 / 9 \mathrm{mmHg}$, which is compatible with other trials of spironolactone as an add-on agent) versus dual RAS blockade (24-hour mean BP reduction of $7 / 3 \mathrm{mmHg}$ ), allowing $53.8 \%$ of patients receiving spironolactone to attain target BP, as opposed to only $25.6 \%$ of those on dual RAS blocker therapy. The results suggest that aldosterone excess plays a bigger role in the pathogenesis of treatment resistance, 


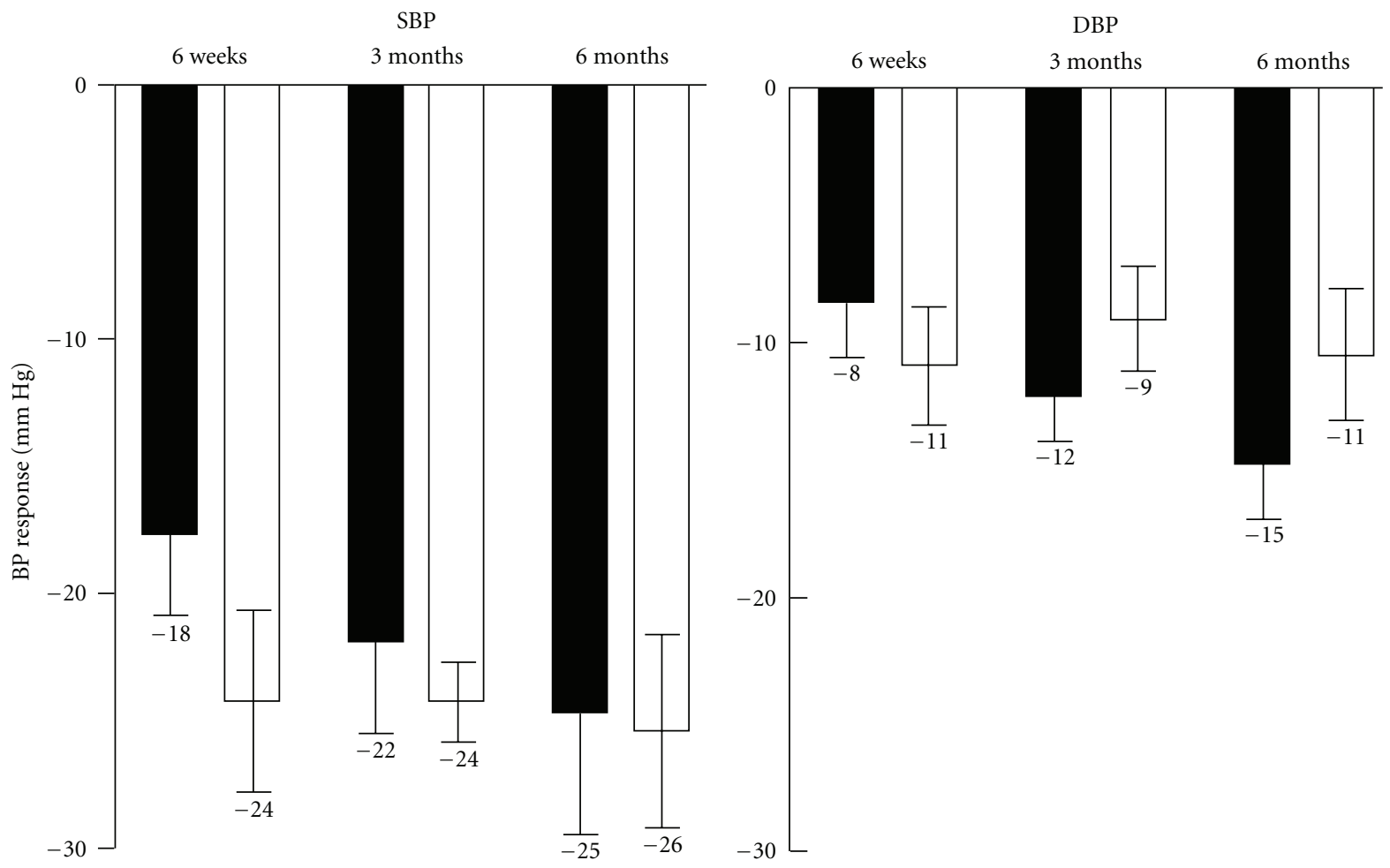

(a)

(b)

FIGURE 1: BP reduction achieved after low-dose spironolactone was added to the antihypertensive regimen in patients with RHTN, with (filled bars) or without (open bars) PA [37].

and that the hypersecretion of aldosterone is relatively autonomous of the RAS, so the magnitude of BP lowering is greater in patients given the MR antagonist compared to those on dual blockade. Further, since the study largely excluded patients with PA, the results show that resistant hypertensive patients in general have an element of relative aldosterone excess, even while measured aldosterone levels fall within the "normal" range.

Some of the more common adverse effects seen with spironolactone treatment are breast tenderness, gynecomastia, erectile dysfunction, and menstrual irregularities, which result from the binding of spironolactone to androgen receptors, preventing its interaction with dihydrotestosterone. The incidence of these adverse effects is rare, reported by about $2-9 \%$ of study patients, and is reversible after discontinuing treatment. Eplerenone, another MR antagonist, binds more selectively to the MR and has a lower affinity for androgen receptors and thus does not have the antiandrogen effects. It is effective in treating patients with RHTN. After receiving eplerenone (at a dose of 50 to $100 \mathrm{mg} /$ day, titrated to achieve $\mathrm{BP}<140 / 90 \mathrm{mmHg}$ ) on top of a three-drug regimen for 12 weeks, office BP was reduced by $18 / 8 \mathrm{mmHg}$, and 24 hour mean BP decreased by $12 / 6 \mathrm{mmHg}(P<.001)$ [40]. Again, these effects were independent of baseline plasma aldosterone and PRA levels.

A head-to-head comparison between spironolactone and eplerenone in patients with primary (not resistant) hypertension and bilateral adrenal hyperplasia showed that the two agents achieved similar degrees of BP lowering in patients with PA [41]. Spironolactone was administered at $400 \mathrm{mg} /$ day (which is a higher dose than usual), while eplerenone was given at $150 \mathrm{mg} /$ day. In spite of the large dose used in the study, only 2 (out of 17) patients who received spironolactone developed painful gynecomastia after 16 weeks of followup, and this was resolved after the patients were shifted to eplerenone. A direct comparison of these two agents in patients with RHTN has not been conducted.

Hyperkalemia can also result from treatment with MR antagonists, particularly in the setting of multidrug therapy that includes RAS blockers, which can also raise serum potassium levels, or in patients with chronic kidney disease. This effect is likewise reversed by discontinuing the MR antagonist or reducing the dose. The incidence of hyperkalemia, however, is low. Out of 76 patients who were given spironolactone for 6 months, only 2 (2.6\%) developed hyperkalemia $(K>5.5 \mathrm{mEq} / \mathrm{L})$ [36]. In patients given eplerenone, the serum potassium increased by a mean of 0.30 $\pm 0.45 \mathrm{mEq} / \mathrm{L}$ after initiation of eplerenone treatment, and mild hyperkalemia (serum potassium 5.5 to $6 \mathrm{mEq} / \mathrm{L}$ ) was seen in only two patients (out of 52 , or $3.8 \%$ ), despite being on an ACE inhibitor or an ARB concomitantly [39].

Beyond BP lowering, treatment with MR antagonists also reverses or attenuates cardiovascular injury mediated by excess aldosterone, particularly the nongenomic effects, 


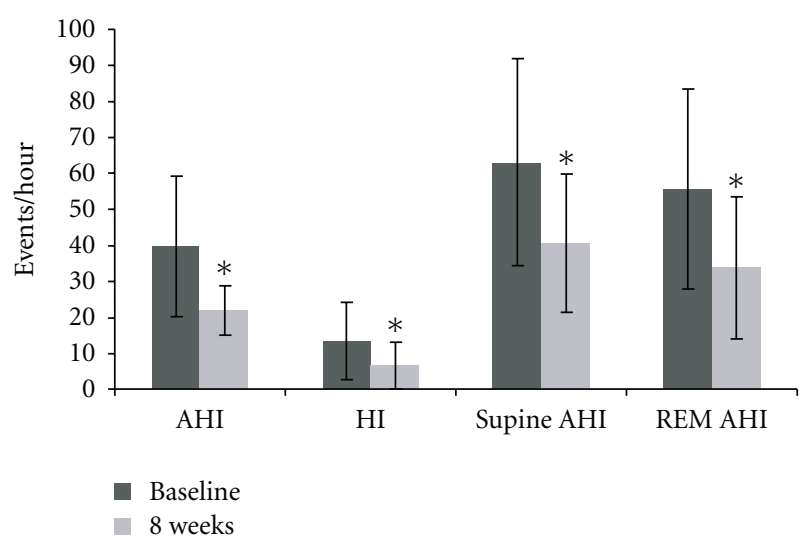

Figure 2: Changes in the apnea-hypopnea index (AHI), hypoxic index (HI), and AHI during supine position or rapid-eye movement (REM) sleep in patients with resistant hypertension before (baseline) and after 8 weeks of add-on spironolactone. ${ }^{*} P<.05$ compared to baseline [44].

which lead to tissue fibrosis, arterial stiffness, and increased oxidative stress. In patients with RHTN (with or without $\mathrm{PA}$ ), spironolactone (at an initial dose of $25 \mathrm{mg}$ /day then forcetitrated to $50 \mathrm{mg} /$ day after 4 weeks) reduced left ventricular mass index at the 3rd and 6th month followup [42]. The degree of LV regression achieved with spironolactone treatment was greater for patients with PA compared to those without PA (22 versus 12\%, resp., $P<.001$ relative to baseline values). Further, spironolactone given to patients with both RHTN and PA for 6 months significantly decreased brain natriuretic peptide values from baseline, an effect that was not seen in those with normal or low aldosterone levels, indicating a prominent diuretic effect even when administered on top of chronic thiazide diuretic treatment. In another study involving patients with RHTN and PA, treatment with low-dose spironolactone ( 12.5 to $25 \mathrm{mg} /$ day) for 3 months significantly increased flow-mediated dilation of the brachial artery, indicating improvement of endothelial function, and this effect was independent of the change in BP [43].

Lastly, spironolactone reduced the severity of OSA in patients with RHTN [44]. In a prospective evaluation involving 12 patients with RHTN in whom spironolactone (25$50 \mathrm{mg} /$ day) was added to a stable antihypertensive regimen, there were significant reductions in the AHI $(39.8 \pm 19.5$ versus $22 \pm 6.8$ events/hour, $P<.05)$, hypoxic index $(13.6 \pm$ 10.8 versus $6.7 \pm 6.6$ events/hour, $P<.05$ ), and clinic and 24 hour ambulatory $\mathrm{BP}$ on the 8 th week followup, and plasma renin activity was increased (Figure 2). Although the study was small and did not have an active control group, this study lends support to the concept that aldosterone excess contributes to the severity of OSA in patients with RHTN.

\section{Summary}

RHTN and PA are each associated with increased cardiovascular risk in hypertensive patients. Aldosterone excess is believed to contribute significantly to uncontrolled BP in patients with RHTN, as shown by a higher prevalence of PA in patients with RHTN and a significant BP-lowering effect produced by MR antagonists, particularly spironolactone, when added to a multidrug antihypertensive regimen that typically already includes a diuretic and an RAS blocker. This effect was seen in both patients with and without PA, showing that even in those patients without elevated aldosterone levels (by current laboratory standards), there is some element of relative aldosterone excess that contributes to the raised BP. Further, treatment with MR antagonists produces cardiovascular benefits beyond $\mathrm{BP}$ lowering (including reduction of the severity of OSA), showing that aldosterone also plays a direct role in the development of target organ damage, arterial stiffness, and endothelial dysfunction, mostly mediated via nongenomic mechanisms.

\section{References}

[1] J. W. Funder, R. M. Carey, C. Fardella et al., "Case detection, diagnosis, and treatment of patients with primary aldosteronism: an endocrine society clinical practice guideline," Journal of Clinical Endocrinology and Metabolism, vol. 93, no. 9, pp. 3266-3281, 2008.

[2] L. M. Fishman, O. Küchel, G. W. Liddle, A. M. Michelakis, R. D. Gordon, and W. T. Chick, "Incidence of primary aldosteronism uncomplicated "essential" hypertension. A prospective study with elevated aldosterone secretion and suppressed plasma renin activity used as diagnostic criteria," Journal of the American Medical Association, vol. 205, no. 7, pp. 497-502, 1968.

[3] N. M. Kaplan, "Hypokalemia in the hypertensive patient, with observations on the incidence of primary aldosteronism," Annals of Internal Medicine, vol. 66, no. 6, pp. 1079-1090, 1967.

[4] A. M. Sinclair, C. G. Isles, and I. Brown, "Seconary hypertension in a blood pressure clinic," Archives of Internal Medicine, vol. 147, no. 7, pp. 1289-1296, 1987.

[5] R. D. Gordon, M. Stowasser, T. J. Tunny, S. A. Klemm, and J. C. Rutherford, "High incidence of primary aldosteronism in 199 patients referred with hypertension," Clinical and Experimental Pharmacology and Physiology, vol. 21, no. 4, pp. 315-318, 1994.

[6] B. L. Rayner, L. H. Opie, and J. S. Davidson, "The aldosterone/renin ratio as a screening test for primary aldosteronism," South African Medical Journal, vol. 90, no. 4, pp. 394400, 2000.

[7] G. P. Rossi, G. Bernini, C. Caliumi et al., "A prospective study of the prevalence of primary aldosteronism in 1,125 hypertensive patients," Journal of the American College of Cardiology, vol. 48, no. 11, pp. 2293-2300, 2006.

[8] L. Mosso, C. Carvajal, A. González et al., "Primary aldosteronism and hypertensive disease," Hypertension, vol. 42, no. 2, pp. 161-165, 2003.

[9] B. Štrauch, T. Zelinka, M. Hampf, R. Bernhardt, and J. Widimsky, "Prevalence of primary hyperaldosteronism in moderate to severe hypertension in the Central Europe region," Journal of Human Hypertension, vol. 17, no. 5, pp. 349-352, 2003.

[10] D. A. Calhoun, D. Jones, S. Textor et al., "Resistant hypertension: siagnosis, evaluation, and treatment a scientific statement from the american heart association professional 
education committee of the council for high blood pressure research," Hypertension, vol. 51, no. 6, pp. 1403-1419, 2008.

[11] S. Douma, K. Petidis, M. Doumas et al., "Prevalence of primary hyperaldosteronism in resistant hypertension: a retrospective observational study," The Lancet, vol. 371, no. 9628, pp. 1921-1926, 2008.

[12] D. A. Calhoun, M. K. Nishizaka, M. A. Zaman, R. B. Thakkar, and P. Weissmann, "Hyperaldosteronism among black and white subjects with resistant hypertension," Hypertension, vol. 40, no. 6, pp. 892-896, 2002.

[13] I. K. Eide, P. A. Torjesen, A. Drolsum, A. Babovic, and N. P. Lilledahl, "Low-renin status in therapy-resistant hypertension: a clue to efficient treatment," Journal of Hypertension, vol. 22, no. 11, pp. 2217-2226, 2004.

[14] B. J. Gallay, S. Ahmad, L. Xu, B. Toivola, and R. C. Davidson, "Screening for primary aldosteronism without discontinuing hypertensive medications: plasma aldosterone-renin ratio," American Journal of Kidney Diseases, vol. 37, no. 4, pp. 699705, 2001.

[15] G. E. Umpierrez, P. Cantey, D. Smiley et al., "Primary Aldosteronism in diabetic subjects with resistant hypertension," Diabetes Care, vol. 30, no. 7, pp. 1699-1703, 2007.

[16] J. W. Funder, "The nongenomic actions of aldosterone," Endocrine Reviews, vol. 26, no. 3, pp. 313-321, 2005.

[17] E. Büssemaker, U. Hillebrand, M. Hausberg, H. Pavenstädt, and H. Oberleithner, "Pathogenesis of hypertension: interactions among sodium, potassium, and aldosterone," American Journal of Kidney Diseases, vol. 55, no. 6, pp. 1111-1120, 2010.

[18] R. S. Vasan, J. C. Evans, M. G. Larson et al., "Serum aldosterone and the incidence of hypertension in nonhypertensive persons," New England Journal of Medicine, vol. 351, no. 1, pp. 33-41, 2004.

[19] G. Bernini, F. Galetta, F. Franzoni et al., "Arterial stiffness, intima-media thickness and carotid artery fibrosis in patients with primary aldosteronism," Journal of Hypertension, vol. 26, no. 12, pp. 2399-2405, 2008.

[20] K. Tsuchiya, T. Yoshimoto, and Y. Hirata, "Endothelial dysfunction is related to aldosterone excess and raised blood pressure," Endocrine Journal, vol. 56, no. 4, pp. 553-559, 2009.

[21] C. Tsioufis, D. Tsiachris, K. Dimitriadis et al., "Myocardial and aortic stiffening in the early course of primary aldosteronism," Clinical Cardiology, vol. 31, no. 9, pp. 431-436, 2008.

[22] G. P. Rossi, G. Bernini, G. Desideri et al., "Renal damage in primary aldosteronism: results of the PAPY study," Hypertension, vol. 48 , no. 2, pp. 232-238, 2006.

[23] P. Milliez, X. Girerd, P. F. Plouin, J. Blacher, M. E. Safar, and J. J. Mourad, "Evidence for an increased rate of cardiovascular events in patients with primary aldosteronism," Journal of the American College of Cardiology, vol. 45, no. 8, pp. 1243-1248, 2005.

[24] C. Catena, G. Colussi, E. Nadalini et al., "Cardiovascular outcomes in patients with primary aldosteronism after treatment," Archives of Internal Medicine, vol. 168, no. 1, pp. 80-85, 2008.

[25] C. McAdam-Marx, X. Ye, J. C. Sung, D. I. Brixner, and K. H. Kahler, "Results of a retrospective, observational pilot study using electronic medical records to assess the prevalence and characteristics of patients with resistant hypertension in an ambulatory care setting," Clinical Therapeutics, vol. 31, no. 5, pp. 1116-1123, 2009.

[26] A. G. Logan, S. M. Perlikowski, A. Mente et al., "High prevalence of unrecognized sleep apnoea in drug-resistant hypertension," Journal of Hypertension, vol. 19, no. 12, pp. 2271-2277, 2001.
[27] C. Cuspidi, G. Macca, L. Sampieri et al., "High prevalence of cardiac and extracardiac target organ damage in refractory hypertension," Journal of Hypertension, vol. 19, no. 11, pp. 2063-2070, 2001.

[28] E. S. Muxfeldt, K. V. Bloch, A. R. Nogueira, and G. F. Salles, "Twenty-four hour ambulatory blood pressure monitoring pattern of resistant hypertension," Blood Pressure Monitoring, vol. 8, no. 5, pp. 181-185, 2003.

[29] S. D. Pierdomenico, D. Lapenna, A. Bucci et al., "Cardiovascular outcome in treated hypertensive patients with responder, masked, false resistant, and true resistant hypertension," American Journal of Hypertension, vol. 18, no. 11, pp. 14221428, 2005.

[30] H. Isaksson and J. Ostergren, "Prognosis in therapy-resistant hypertension," Journal of Internal Medicine, vol. 236, no. 6, pp. 643-649, 1994.

[31] F. Javier Nieto, T. B. Young, B. K. Lind et al., "Association of sleep-disordered breathing sleep apnea, and hypertension in a large community-based study," Journal of the American Medical Association, vol. 283, no. 14, pp. 1829-1836, 2000.

[32] M. N. Pratt-Ubunama, M. K. Nishizaka, R. L. Boedefeld, S. S. Cofield, S. M. Harding, and D. A. Calhoun, "Plasma aldosterone is related to severity of obstructive sleep apnea in subjects with resistant hypertension," Chest, vol. 131, no. 2, pp. 453-459, 2007.

[33] P. Lavie and V. Hoffstein, "Sleep apnea syndrome: a possible contributing factor to resistant," Sleep, vol. 24, no. 6, pp. 721725, 2001.

[34] T. A. Dernaika, G. T. Kinasewitz, and M. M. Tawk, "Effects of nocturnal continuous positive airway pressure therapy in patients with resistant hypertension and obstructive sleep apnea," Journal of Clinical Sleep Medicine, vol. 5, no. 2, pp. 103107, 2009.

[35] L. Lozano, J. L. Tovar, G. Sampol et al., "Continuous positive airway pressure treatment in sleep apnea patients with resistant hypertension: a randomized, controlled trial," Journal of Hypertension, vol. 28, no. 10, pp. 2161-2168, 2010.

[36] A. Di Murro, L. Petramala, D. Cotesta et al., "Reninangiotensin-aldosterone system in patients with sleep apnoea: prevalence of primary aldosteronism," Journal of the ReninAngiotensin-Aldosterone System, vol. 11, no. 3, pp. 165-172, 2010.

[37] M. K. Nishizaka, M. A. Zaman, and D. A. Calhoun, "Efficacy of low-dose spironolactone in subjects with resistant hypertension," American Journal of Hypertension, vol. 16, no. 11, pp. 925-930, 2003.

[38] F. De Souza, E. Muxfeldt, R. Fiszman, and G. Salles, "Efficacy of spironolactone therapy in patients with true resistant hypertension," Hypertension, vol. 55, no. 1, pp. 147-152, 2010.

[39] B. Alvarez-Alvarez, M. Abad-Cardiel, A. Fernandez-Cruz, and N. Martell-Claros, "Management of resistant arterial hypertension: role of spironolactone versus double blockade of the renin-angiotensin-aldosterone system," Journal of Hypertension, vol. 28, no. 11, pp. 2329-2335, 2010.

[40] D. A. Calhoun and W. B. White, "Effectiveness of the selective aldosterone blocker, eplerenone, in patients with resistant hypertension," Journal of the American Society of Hypertension, vol. 2, no. 6, pp. 462-468, 2008.

[41] A. Karagiannis, K. Tziomalos, A. Papageorgiou et al., "Spironolactone versus eplerenone for the treatment of idiopathic hyperaldosteronism," Expert Opinion on Pharmacotherapy, vol. 9, no. 4, pp. 509-515, 2008.

[42] K. Gaddam, C. Corros, E. Pimenta et al., "Rapid reversal of left ventricular hypertrophy and intracardiac volume overload in 
patients with resistant hypertension and hyperaldosteronism: a prospective clinical study," Hypertension, vol. 55, no. 5, pp. 1137-1142, 2010.

[43] M. K. Nishizaka, M. A. Zaman, S. A. Green, K. Y. Renfroe, and D. A. Calhoun, "Impaired endothelium-dependent flowmediated vasodilation in hypertensive subjects with hyperaldosteronism," Circulation, vol. 109, no. 23, pp. 2857-2861, 2004.

[44] K. Gaddam, E. Pimenta, S. J. Thomas et al., "Spironolactone reduces severity of obstructive sleep apnoea in patients with resistant hypertension: a preliminary report," Journal of Human Hypertension, vol. 24, no. 8, pp. 532-537, 2009. 


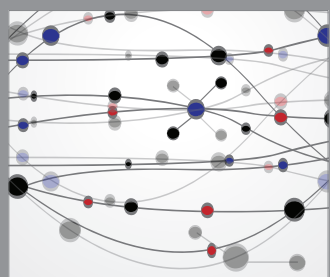

The Scientific World Journal
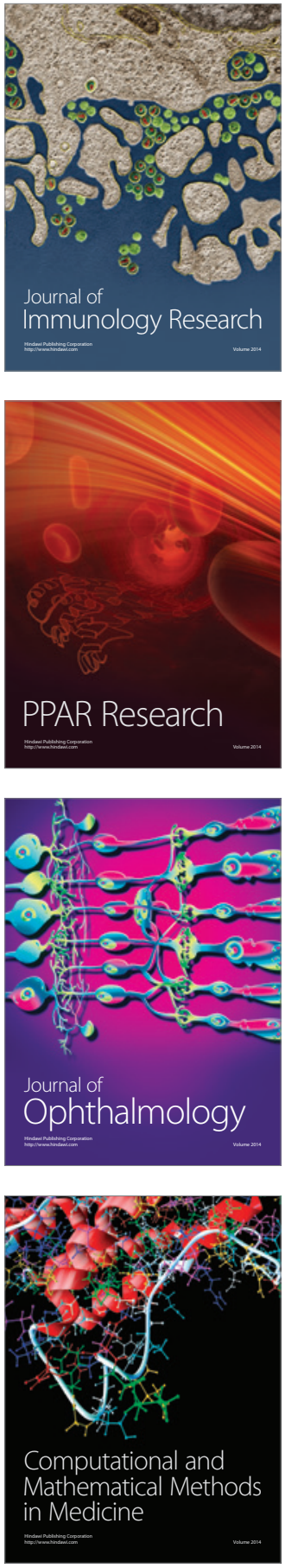

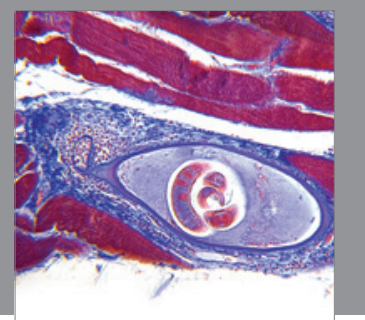

Gastroenterology

Research and Practice
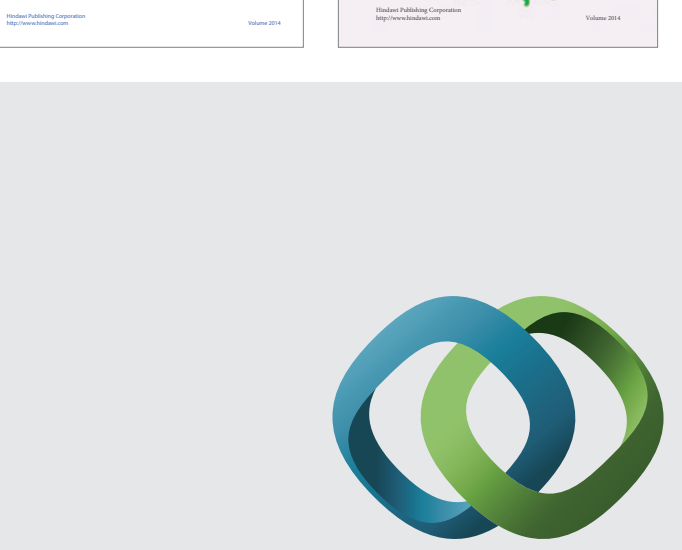

\section{Hindawi}

Submit your manuscripts at

http://www.hindawi.com
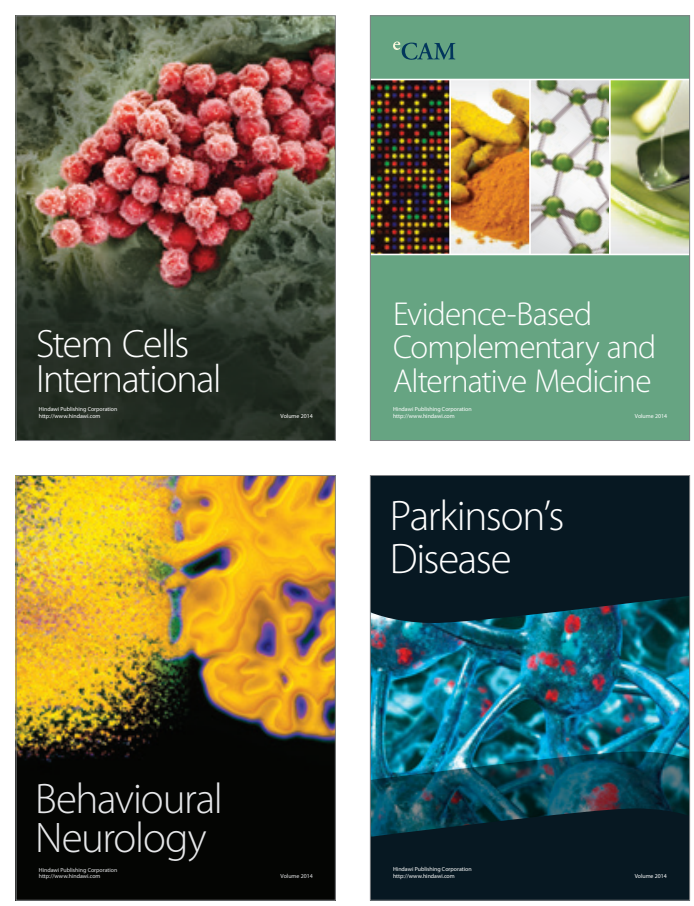

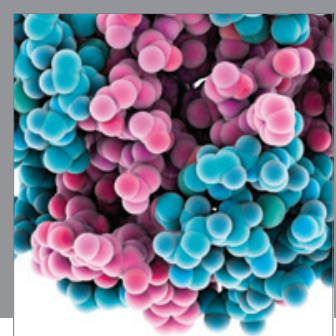

Journal of
Diabetes Research

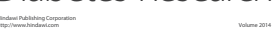

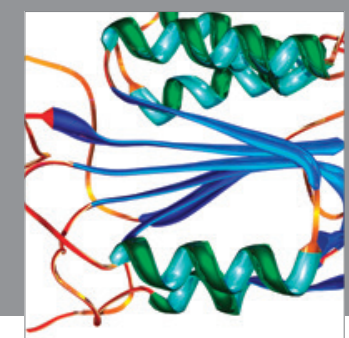

Disease Markers
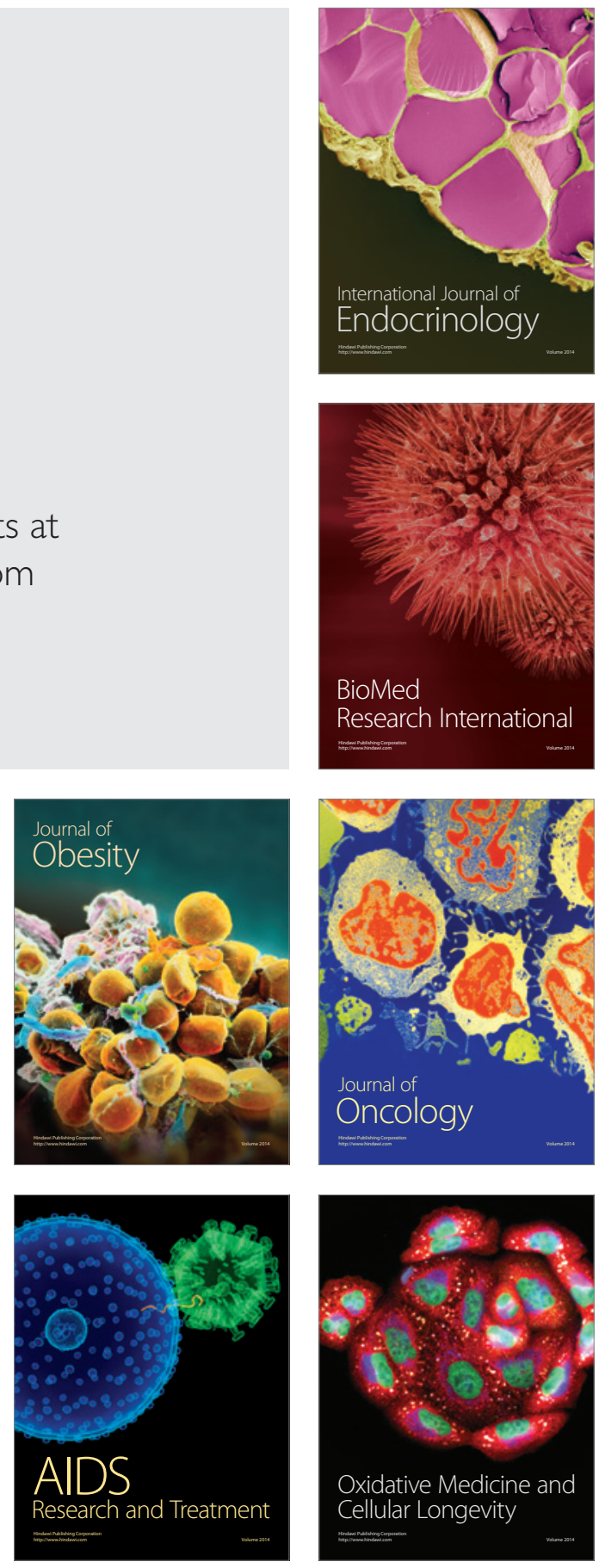